\title{
Elongational viscosity scaling of polymer melts with different chemical constituents
}

\author{
Esmaeil Narimissa ${ }^{1,2} \cdot$ Leslie Poh $^{1,2} \cdot$ Manfred H. Wagner $^{3}$ (D) \\ Received: 29 October 2020 / Revised: 23 January 2021 / Accepted: 1 February 2021 / Published online: 4 March 2021 \\ (C) The Author(s) 2021
}

\begin{abstract}
Morelly et al. (Macromolecules 52:915-922, 2019) reported transient and steady-state elongational viscosity data of monodisperse linear polymer melts obtained by filament-stretching rheometry with locally controlled strain and strain rate and found different power law scaling of the elongational viscosities of polystyrene, poly(tert-butylstyrene) and poly(methyl-methacrylate). Very good agreement is achieved between data and predictions of the extended interchain pressure (EIP) model (Narimissa et al. J. Rheol. 64, 95-110 (2020)), based solely on linear viscoelastic characterization and the Rouse time $\tau_{R}$ of the melts. The analysis reveals that both the normalized elongational viscosity and the normalized elongational stress are dependent on the number of entanglements $(Z)$ and the ratio of entanglement molar mass $M_{\mathrm{em}}$ to critical molar mass $M_{\mathrm{cm}}$ of the melts in the linear viscoelastic regime through $\eta_{E}^{0} /\left(G_{N} \tau_{R}\right) \propto\left(M_{\mathrm{em}} / M_{\mathrm{cm}}\right){ }^{2.4} Z^{1.4}$ and $\sigma_{E}^{0} / G_{N} \propto\left(M_{\mathrm{em}} / M_{\mathrm{cm}}\right){ }^{2.4} Z^{1.4} W i$, while in the limit of fast elongational flow with high Weissenberg number $W i=\tau_{R} \dot{\varepsilon}$, both viscosity and stress become independent of $Z$ and $M_{\mathrm{em}} / M_{\mathrm{cm}}$, and approach a scaling which depends only on $W i$, i.e. $\eta_{E} /\left(G_{N} \tau_{R}\right) \propto W i^{-1 / 2}$ and $\sigma_{E} / G_{N} \propto W i^{1 / 2}$. When expressed by an effective power law, the broad transition from the linear viscoelastic to the high $W i$ regime leads to chemistry-dependent scaling at intermediate $W i$ depending on the number of entanglements and the ratio between entanglement molar mass and critical molar mass.
\end{abstract}

Keywords EIP model $\cdot$ Elongation $\cdot$ Monodisperse melt $\cdot$ Polystyrene $\cdot$ Poly(tert-butylstyrene $) \cdot$ Poly(methyl-methacrylate) Universality

\section{Introduction}

Decades long of intense research have led to the development of fundamental theories (Flory 1953; Kuhn 1934; Rouse Jr 1953; Zimm 1956) of polymer rheology, with the tube model of de Gennes (Gennes 1971) and Doi and Edwards (Doi and Edwards 1979; Doi and Edwards 1986) establishing a fundamental basis for explaining the dynamics of entangled

Esmaeil Narimissa

esmaeiln@technion.ac.il

$\triangle$ Manfred H. Wagner

manfred.wagner@tu-berlin.de

1 Dept. of Chemical Engineering, Technion-Israel Institute of Technology (IIT), Technion City, 32000 Haifa, Israel

2 Dept. of Chemical Engineering, Guangdong Technion-Israel Institute of Technology (GTIIT), Shantou 515063, China

3 Polymer Engineering/Polymer Physics, Berlin Institute of Technology (TU Berlin), Ernst-Reuter-Platz 1, 10587 Berlin, Germany polymers. However, as new discoveries are being made through the development of new testing methods and the release of new rheological data, this may challenge existing well-established concepts and calls for the development of new concepts or the improvement of existing models. Experimental studies over the past decades have demonstrated several insufficiencies of the tube model and called for modifications in modelling the linear and nonlinear viscoelastic properties of polymers. Some of the early advancements made were the introduction of the concepts of constraint release (CR) (Daoud and De Gennes 1979) and contour length fluctuations (CLF) (Doi 1981) for linear entangled polymer melts and solutions in the linear viscoelastic (LVE) regime, as well as chain stretch mechanisms (Marrucci and Grizzuti 1988) and convective constraint release (CCR) (Ianniruberto and Marrucci 1996), to account for nonlinear viscoelasticity of entangled polymers in extensional flows (for a recent review, see, e.g. Narimissa and Wagner (2019)).

While there seems to be general agreement on the universality of the linear viscoelastic behaviour of well-entangled monodisperse linear polymer melts and solutions based on 
only three material parameters (plateau modulus, characteristic time and the number of entanglements), this universality is lost in the nonlinear viscoelastic regime as especially apparent in elongational flow, where not only differences in the rheology of melts and solutions became apparent but also different signatures of different chemistries are reported (Morelly et al. 2019). However, we note that Fetters et al. (1999b) collected data for a large number of polymers and showed that the ratio of the entanglement molar mass $M_{\mathrm{em}}$ to the critical molar mass of $M_{\mathrm{cm}}$ is chemistry dependent. They concluded that the degree of entanglement as specified by $M_{\mathrm{em}}$ is not sufficient to fully characterize the entanglement effects. Thus, the three material parameters plateau modulus, characteristic time and number of entanglements may not be sufficient to fully characterize the apparent universality of the linear viscoelastic behaviour of well-entangled monodisperse linear polymer melts and solutions.

With the development of the filament-stretching rheometer (FSR) (Bach et al. 2003b), a key experimental study by Bach et al. (2003a) showed that there is a monotonic decrease in the elongational viscosity $\eta_{E}$ even at strain rates larger than the inverse of the Rouse time $\dot{\varepsilon}>\tau_{R}{ }^{-1}$ in polystyrene (PS) melts. This observation was different from what was expected by the original Doi-Edwards model and what was found experimentally for PS solutions by Bhattacharjee et al. (2002) and Ye et al. (2003), i.e. the monotonic decrease in $\eta_{E}$ is followed by a sudden increase at $\dot{\varepsilon} \tau_{R}{ }^{-1}$ when chain stretch is supposed to set in. Yaoita et al. (2012) brought forth the concept that the monomeric friction coefficient may decrease due to flow-induced monomer coalignment and used this to explain the observation made on monotonic extension thinning of polymer melts. By performing nonequilibrium molecular dynamics simulation of fast shear flows of oligomeric polystyrene chains, Ianniruberto et al. (2012) found evidence that the monomeric friction coefficient decreases in fast elongational flows. To explain the observed discrepancies between melts and solutions, Desai and Larson (2014) tested the influence of Kuhn segment alignment on local friction based on the hypothesis of Yaoita et al. (2012) that the monomeric friction coefficient is dependent on a stretch/orientation parameter. However, Huang and coworkers performed a sequence of experiments on PS diluted in oligomeric styrene with the same number of entanglements, but different polymer concentrations (Huang et al. 2015) and different solvent molar masses (Huang et al. 2013a). These studies observed different normalized viscosities at high strain rates for polymers with a similar concentration in different solvent molar masses. Therefore, the cause for the observed differences between melts and solutions cannot be exclusively found in the monomeric friction reduction concept. Huang et al. (2013a) hypothesized that nematic interactions between polymer-solvent and polymer-polymer molecules may be partially responsible for the nonlinear rheological behaviour of concentrated polymer solutions and melts in strong extensional flows. Based on these elongational viscosity data and the hypothesis of Huang et al. (2013a), Ianniruberto (Ianniruberto 2015) and Park and Ianniruberto (Park and Ianniruberto 2017) proposed a flow-induced monomeric friction reduction-based multimode model that also takes into account nematic interactions. A recent review by Ianniruberto et al. (2020) claims "the plausible certainty" that flow-induced coalignment of the Kuhn segments of the polymer modifies the friction coefficient, which may depart considerably from the equilibrium value and may be sensitive to the chemistry, being different in different polymers. The authors conclude that the universality of the rheological behaviour of polymer melts, which is well documented under equilibrium conditions and in relatively slow flows, is lost in fast flows.

However, already in 2004, the set of experimental data by Bach et al. (2003a) has led Marrucci and Ianniruberto (2004) to suggest an interchain pressure (IP) model to rationalize the unexpected extension thinning at elongation rates above the inverse Rouse time of polymer melts. Starting from this concept, Wagner et al. (2005) developed an extended interchain pressure (EIP) model by correlating the Rouse stretch relaxation time and the tube diameter relaxation time suggested by Marrucci and Ianniruberto (2004). Using the EIP model, Narimissa et al. (2020a) considered the dependence of the interchain tube pressure effect on polymer concentration and molar mass of oligomeric solvents, and they demonstrated good agreement between model predictions and the elongational viscosity data of Bach et al. (2003a) for polystyrene melts and Huang et al. (2013a, 2015, 2013b) for polystyrene solutions. In addition, a novel constitutive model was proposed by Narimissa et al. (2020b) consisting of a combination of the EIP model and the Doi-Edwards independent alignment (DEIA) concept to address the overprediction of the steady-state shear viscosity and the steady-state first normal stress coefficient at high shear rates.

It should be noted that the EIP model is based on the LVE characterization of melts and solutions and the Rouse time, and its universality in modelling the nonlinear viscoelasticity of polystyrene melts and solutions of different molar masses and concentrations has been well documented. It is therefore of interest to test the applicability of the EIP model to the elongational viscosity data of monodisperse linear polymer melts other than polystyrene and to see whether universality of the model in the sense discussed above, i.e. based exclusively on the LVE characterization and the Rouse time, is maintained also for polymer melts with different chemical constituents. Recently, Morelly et al. (2019) compared the nonlinear elongational viscosity behaviour of polystyrene (PS) with melts of poly(tert-butylstyrene) (PtBS) and poly(methyl-methacrylate) (PMMA). While the normalized 
LVE of monodisperse linear polymer melts is exclusively dependent on the number of entanglements, they found different elongational scaling of the elongational viscosity for different chemistries. In this article, we analyse the elongational viscosity data of well-defined entangled polymer melts by Bach et al. (2003a), Huang et al. (2013b) and Morelly et al. (2019), obtained by the filament-stretching rheometer with local strain and strain rate control and compare data with the predictions of the EIP model for nonlinear elongational rheology of monodisperse entangled polymer melts.

The article is organized as follows: In the "Experimental data and LVE characterization" section, we shortly introduce the polymers examined in this paper and the experimental methods used to determine LVE and elongational viscosity, followed by a presentation of the linear viscoelastic characterization of the polymers of this study. "The extended interchain pressure model" section gives a summary of the modelling approach of the extended interchain pressure (EIP) model. Experimental data and model predictions of the EIP model are compared in the "Comparison between experimental data and model predictions" section, followed by discussion and conclusions in the "Discussion and conclusions" section.

\section{Experimental data and LVE characterization}

\section{Materials}

The nonlinear elongational stress data of polystyrene (PS), polytert-butylstyrene (PtBS) and polymethyl-methacrylate (PMMA) considered in this study were taken from various pieces of literature (Bach et al. 2003a; Huang et al. 2013b; Morelly et al. 2019). The PS samples (PS200k, PS-285k) and the PtBS sample (PtBS-301k) were prepared by anionic polymerization. PMMA samples (PMMA-86k and PMMA-100k) were purchased from Polymer Standards Service GmbH and used as received (Morelly et al. 2019). The molecular characteristics of the polymer melts are summarized in Table 1.

The linear viscoelastic properties of the polystyrene (PS), poly(methyl-methacrylate) (PMMA) and poly(tertbutylstyrene) (PtBS) melts were obtained from small amplitude oscillatory shear (SAOS) flow measurements. Measurements for PS-200k were performed using parallelplate geometry on an AR2000 rheometer from TA Instruments at 130 and $150{ }^{\circ} \mathrm{C}$ (Bach et al. 2003a, Huang et al. 2013b, Morelly et al. 2019). Measurements for PS$285 \mathrm{k}$ were performed using $88-\mathrm{mm}$ parallel-plate geometry on an ARES-G2 rheometer from TA Instruments at 130, 150 and $170{ }^{\circ} \mathrm{C}$ under nitrogen (Bach et al. 2003a, Huang et al. 2013b, Morelly et al. 2019). The measurements for PtBS and PMMA samples were performed using 8- or 25-mm parallelplate geometry on a DHR-3 rheometer (TA Instruments) with electrically heated plates (EHP) at $175,180,190$ and $200^{\circ} \mathrm{C}$ in the air (PtBS-301k) and at $140,150,170$ and $190^{\circ} \mathrm{C}$ under nitrogen (PMMA-86k and PMMA-100k) (Bach et al. 2003a, Huang et al. 2013b, Morelly et al. 2019). The linear viscoelastic data were shifted to a single master curve at $130{ }^{\circ} \mathrm{C}$ for PS, at $150{ }^{\circ} \mathrm{C}$ for PMMA and at $175{ }^{\circ} \mathrm{C}$ for PtBS using the time temperature superposition (TTS) procedure.

Elongational stress measurements for both PS melts was performed using a homemade filament-stretching rheometer (FSR) equipped with an oven to allow measurements from room temperature to elevated temperatures (Bach et al. 2003b), while the measurements for PMMA and PtBS were performed using a commercial filament stretch rheometer VADER 1000 (Rheo Filament ApS, Albertslund, DK) (Bach et al. 2003a, Huang et al. 2013b, Morelly et al. 2019). The PS200k samples were pressed at $150{ }^{\circ} \mathrm{C}$ for about $2 \mathrm{~min}$ with temperatures chosen as low as possible to prevent degradation while still being high enough for stress relaxation (Bach et al. 2003a, Huang et al. 2013b, Morelly et al. 2019). The PS-285k samples were pressed at approximately $150{ }^{\circ} \mathrm{C}$ (Bach et al. 2003a, Huang et al. 2013b, Morelly et al. 2019), the PtBS samples at 200 or $220{ }^{\circ} \mathrm{C}$ and the PMMA samples at $190{ }^{\circ} \mathrm{C}$ (Bach et al. 2003a, Huang et al. 2013b, Morelly et al. 2019). The polymer melts were annealed at the temperatures mentioned for $15 \mathrm{~min}$ under vacuum to ensure that the polymers had sufficient time to relax. The polymers were checked for degradation after the moulding by size exclusion chromatography.

\section{LVE characterization}

From linear viscoelastic master curves of $\mathrm{G}^{\prime}$ and $\mathrm{G}^{\prime \prime}$, continuous Baumgärtel-Schausberger-Winter (BSW) relaxation spectra (Baumgaertel et al. 1990) were determined by Huang et al. (2013a, 2015, 2013b) and by Morelly et al. (2019) and used in their analysis of the data. The relaxation modulus $G(t)$ is found from the spectrum $H(\tau)$ by

$G(t)=\int_{0}^{\infty} \frac{H(\tau)}{\tau} \exp (-t / \tau) d \tau$

$H(\tau)$ is composed of two parts, the entanglement contribution $H_{e}(\tau)$ and the glassy contribution $H_{g}(\tau)$,

$H(\tau)=H_{e}(\tau)+H_{g}(\tau)$

with

$H_{e}(\tau)=n_{e} G_{N}\left(\frac{\tau}{\tau_{t}}\right)^{n_{e}} h\left(1-\tau / \tau_{t}\right)$ 
Table 1 Molecular

characterization, test temperature

(T) and material parameters of

PtBS-310k (Morelly et al. 2019),

PMMA-86k (Morelly et al.

2019), PMMA-100k (Morelly

et al. 2019), PS-200k (Bach et al.

2003a) and PS-285k (Huang et al.

2013a) from linear viscoelastic

data using IRIS (Winter and

Mours 2006) spectra of Table 2

\begin{tabular}{llllll}
\hline & PtBS-301k & PMMA-86k & PMMA-100k & PS-200K & PS-285K \\
\hline$M(\mathrm{~kg} / \mathrm{mol})$ & 301 & 86 & 99.4 & 200 & 285 \\
$M / M_{n}$ & 1.02 & 1.02 & 1.08 & 1.04 & 1.09 \\
$\rho\left(\mathrm{kg} / \mathrm{m}^{3}\right)$ & 957 & 1180 & 1180 & 1000 & 1000 \\
$G_{N}(\mathrm{kPa})$ & 120 & 720 & 850 & 250 & 250 \\
$T\left({ }^{\circ} \mathrm{C}\right)$ & 175 & 150 & 160 & 130 & 128 \\
$\tau_{d}(\mathrm{~s})$ & 3225 & 952 & 297 & 961 & 8643 \\
$\eta_{0}(\mathrm{MPa} \cdot \mathrm{s})$ & 95.5 & 121 & 56.5 & 82.6 & 531 \\
$M_{\mathrm{em}}(\mathrm{kg} / \mathrm{mol})$ & 30 & 5.8 & 5.0 & 13.4 & 13.3 \\
$Z$ & 10.1 & 14.9 & 19.9 & 14.9 & 21.4 \\
$N_{\varepsilon}$ & 29 & 8 & 7 & 16 & 16 \\
$M_{\mathrm{cm}}(\mathrm{kg} / \mathrm{mol})$ & 95 & 9.2 & 8.0 & 35 & 35 \\
$\tau_{R}(\mathrm{~s})$ & $\left.618(306)^{*}\right)$ & $\left.14.4(37.7)^{*}\right)$ & $\left.3.80(12.5)^{*}\right)$ & $\left.91.5(90.0)^{*}\right)$ & 360 \\
$T_{g}\left({ }^{\circ} \mathrm{C}\right)$ & - & 122.8 & - & - & 107.5 \\
\hline
\end{tabular}

${ }^{*)} \tau_{R}$ is calculated by Eq. (14) (Morelly et al. 2019) and

$H_{g}(\tau)=n_{e} G_{N}\left(\frac{\tau}{\tau_{c}}\right)^{-n_{g}} h\left(1-\tau / \tau_{t}\right)$

$G_{N}$ is the plateau modulus, $\tau_{t}$ the longest or "terminal" relaxation time, $\tau_{c}$ the characteristic time constant of the glassy contribution (related to the Kuhn segment equilibration time $\left.\tau_{e}\right)$ and $h(x)$ the Heaviside step function. The values of $n_{e}$ and $n_{g}$ were fixed to 0.23 and 0.70 , respectively (Huang et al. 2013a, 2013b). The physical meaning of the BSW parameters can be found elsewhere (Huang et al. 2013b), and the BSW parameters of the polymer melts considered are given in Morelly et al. (2019).

From the BSW spectra, the plateau modulus $G_{N}$ of the samples is obtained, and from $G_{N}$, the entanglement molar mass $M_{\mathrm{em}}$ of the melt is calculated as (Huang et al. 2013b)

$M_{\mathrm{em}}=\frac{\rho R T}{G_{N}}$

where $\rho$ is the density of polymer melt at the testing temperature, $R$ is the gas constant and $T$ the absolute temperature. With $M$ being the molar mass of the polymer, the number of entanglements per chain, $Z$, is obtained,

$Z=\frac{M}{M_{\mathrm{em}}}$

The number of Kuhn segments between entanglements is,

$N_{e}=\frac{M_{\mathrm{em}}}{M_{0}}$ where $M_{0}$ is the molar mass of the Kuhn segment. Values for $M_{\mathrm{em}}$ and $M_{0}$ are given in Morelly et al. (2019).

While the BSW spectrum defined by Eqs. (1) to (4) allows for a reasonable quantification of the plateau modulus $G_{N}$, we will show in the "Comparison between experimental data and model predictions" section that the BSW spectrum derived by Morelly et al. (2019) does not result in an ideal fit of the $G^{\prime}$ and $G^{\prime \prime}$ master curves of PtBS-301k, PMMA-86k and PMMA$100 \mathrm{k}$. We will therefore use parsimonious relaxation spectra,

$G(t)=\sum_{i=1} g_{i} \exp \left(-t / \tau_{i}\right)$

for characterization of LVE in the experimentally accessible frequency window of all polymers considered here. The partial moduli $g_{i}$ and relaxation times $\tau_{i}$ as computed by the IRIS software (Winter and Mours 2006) are presented in Table 2 and result in excellent agreement with the linear viscoelastic data of $G^{\prime}$ and $G^{\prime \prime}$. We note that any quantitative representation of LVE can be used in the EIP model, either obtained from "molecular" or mesoscopic modelling, or directly from the experimental data, e.g. by parsimonious spectra as used here.

According to the DE model, the Rouse time $\tau_{R}$, the disengagement (or reptation) time $\tau_{d}$ and the zero-shear viscosity $\eta_{0}$ are given by (see e.g. Dealy et al. (2018)),

$$
\begin{aligned}
\tau_{R} & =Z^{2} \tau_{e} \\
\tau_{d} & =3 Z \tau_{R} \\
\eta_{0} & =\frac{\pi^{2}}{12} G_{N} \tau_{d}
\end{aligned}
$$

Here, we identify $\tau_{d}$ with the mean quadratic average of the relaxation times of the discrete relaxation spectrum and calculate $\eta_{0}$ from the discrete relaxation spectrum, 
Table 2 Discrete relaxation spectra of PtBS-310k (at $T=175^{\circ} \mathrm{C}$ (Morelly et al. 2019)), PMMA-86k (at $T=150{ }^{\circ} \mathrm{C}$ (Morelly et al. 2019)), PMMA$100 \mathrm{k}$ (at $T=160{ }^{\circ} \mathrm{C}$ (Morelly et al. 2019)), PS-200k (at $T=130^{\circ} \mathrm{C}$ ) (Bach et al. 2003a) and PS-285k (at $T=128^{\circ} \mathrm{C}$ ) (Huang et al. 2013a)

\begin{tabular}{|c|c|c|c|c|c|c|c|c|c|}
\hline \multicolumn{2}{|c|}{ PMMA-86k } & \multicolumn{2}{|l|}{ PtBS-310k } & \multicolumn{2}{|c|}{ PMMA-100k } & \multicolumn{2}{|l|}{ PS-200k } & \multicolumn{2}{|l|}{ PS-285k } \\
\hline$g_{i}(\mathrm{~Pa})$ & $\tau_{i}(\mathrm{~s})$ & $g_{i}(\mathrm{~Pa})$ & $\tau_{i}(\mathrm{~s})$ & $g_{i}(\mathrm{~Pa})$ & $\tau_{i}(\mathrm{~s})$ & $g_{i}(\mathrm{~Pa})$ & $\tau_{i}(\mathrm{~s})$ & $g_{i}(\mathrm{~Pa})$ & $\tau_{i}(\mathrm{~s})$ \\
\hline $5.88 \mathrm{E}+07$ & $5.00 \mathrm{E}-04$ & $9.21 \mathrm{E}+07$ & $4.65 \mathrm{E}-04$ & $2.80 \mathrm{E}+08$ & $1.33 \mathrm{E}-05$ & $2.38 \mathrm{E}+05$ & $4.75 \mathrm{E}-01$ & 4.34E+08 & 7.76E-05 \\
\hline $5.94 \mathrm{E}+06$ & $3.66 \mathrm{E}-03$ & $1.32 \mathrm{E}+06$ & $1.21 \mathrm{E}-02$ & $3.57 \mathrm{E}+06$ & $8.99 \mathrm{E}-04$ & $3.71 \mathrm{E}+04$ & $5.29 \mathrm{E}+00$ & $1.93 \mathrm{E}+06$ & $7.86 \mathrm{E}-03$ \\
\hline $1.31 \mathrm{E}+06$ & $2.12 \mathrm{E}-02$ & $4.79 \mathrm{E}+05$ & $6.34 \mathrm{E}-02$ & $9.31 \mathrm{E}+05$ & $4.79 \mathrm{E}-03$ & $2.71 \mathrm{E}+04$ & $1.96 \mathrm{E}+01$ & $5.61 \mathrm{E}+05$ & 4.60E-02 \\
\hline $5.52 \mathrm{E}+05$ & $8.42 \mathrm{E}-02$ & $2.06 \mathrm{E}+05$ & $3.14 \mathrm{E}-01$ & $3.32 \mathrm{E}+05$ & $2.19 \mathrm{E}-02$ & $5.46 \mathrm{E}+04$ & $9.20 \mathrm{E}+01$ & $2.33 \mathrm{E}+05$ & $2.26 \mathrm{E}-01$ \\
\hline $2.31 \mathrm{E}+05$ & $3.70 \mathrm{E}-01$ & $8.33 \mathrm{E}+04$ & $1.65 \mathrm{E}+00$ & $1.49 \mathrm{E}+05$ & $9.63 \mathrm{E}-02$ & $4.96 \mathrm{E}+04$ & $4.43 \mathrm{E}+02$ & $9.88 \mathrm{E}+04$ & $1.20 \mathrm{E}+0 \mathrm{C}$ \\
\hline $1.50 \mathrm{E}+05$ & $1.74 \mathrm{E}+00$ & $3.49 \mathrm{E}+04$ & $5.96 \mathrm{E}+00$ & $1.53 \mathrm{E}+05$ & 4.67E-01 & 4.33E+04 & $1.26 \mathrm{E}+03$ & 4.43E+04 & $6.92 \mathrm{E}+0 \mathrm{C}$ \\
\hline $1.76 \mathrm{E}+05$ & $8.11 \mathrm{E}+00$ & $3.48 \mathrm{E}+04$ & $2.42 \mathrm{E}+01$ & $1.77 \mathrm{E}+05$ & $2.34 \mathrm{E}+00$ & - & - & $3.23 \mathrm{E}+04$ & $3.63 \mathrm{E}+01$ \\
\hline $1.99 \mathrm{E}+05$ & $4.01 \mathrm{E}+01$ & $3.24 \mathrm{E}+04$ & $1.33 \mathrm{E}+02$ & $1.81 \mathrm{E}+05$ & $1.26 \mathrm{E}+01$ & - & - & $3.23 \mathrm{E}+04$ & $1.45 \mathrm{E}+02$ \\
\hline $2.04 \mathrm{E}+05$ & $1.87 \mathrm{E}+02$ & $2.69 \mathrm{E}+04$ & $6.70 \mathrm{E}+02$ & $1.91 \mathrm{E}+05$ & $5.76 \mathrm{E}+01$ & - & - & $4.04 \mathrm{E}+04$ & $5.42 \mathrm{E}+02$ \\
\hline $1.27 \mathrm{E}+05$ & $4.96 \mathrm{E}+02$ & $1.60 \mathrm{E}+04$ & $2.87 \mathrm{E}+03$ & $1.70 \mathrm{E}+05$ & $1.96 \mathrm{E}+02$ & - & - & $4.17 \mathrm{E}+04$ & $2.07 \mathrm{E}+03$ \\
\hline $1.41 \mathrm{E}+03$ & $7.38 \mathrm{E}+03$ & $4.11 \mathrm{E}+03$ & $6.31 \mathrm{E}+03$ & $9.37 \mathrm{E}+03$ & $1.01 \mathrm{E}+03$ & - & - & $5.53 \mathrm{E}+04$ & $6.83 \mathrm{E}+03$ \\
\hline- & - & - & - & - & - & - & - & $8.44 \mathrm{E}+02$ & $4.65 \mathrm{E}+04$ \\
\hline
\end{tabular}

$$
\begin{array}{r}
\tau_{d}=\frac{\sum_{i} g_{i} \tau_{i}^{2}}{\sum_{i} g_{i} \tau_{i}} \\
\eta_{0}=\sum_{i} g_{i} \tau_{i}
\end{array}
$$

We note that Morelly et al. (2019) assumed the equivalence of $\tau_{e}$ and $\tau_{c}$, i.e. $\tau_{e} \cong \tau_{c}$, and calculated the Rouse time in correspondence with Eq. (9) by the approximation

$\tau_{R} \cong Z^{2} \tau_{c}$

We prefer to use Osaki's approach (Isaki et al. 2003; Menezes and Graessley 1982; Osaki et al. 1982; Takahashi et al. 1993) for quantification of the Rouse time $\tau_{R}$, which extrapolates the Rouse time of unentangled polymer systems to the Rouse time of entangled polymer melts and takes into account the power of 3.4 scalings of the zero-shear viscosity with the molar mass $M$. This leads to the relation,

$\tau_{R}=\frac{12 M \eta_{0}}{\pi^{2} \rho R T}\left(\frac{M_{\mathrm{cm}}}{M}\right)^{2.4}$

$M_{\mathrm{cm}}$ denotes the critical molar mass in the melt state. For monodisperse polystyrene, we use the well-documented value of $M_{\mathrm{cm}}=35 \mathrm{~kg} / \mathrm{mol}$ (Ferry 1980; Luap et al. 2005; Wagner 2014), and Eq. (15) has been used successfully for modelling of the transient and steady-state elongational and shear viscosities of PS melts and solutions (Narimissa et al. 2020a, b). In the case of PtBS and PMMA, the monomeric friction coefficient $\zeta$ and subsequently the equilibration time $\tau_{e}$ are unknown; hence, a realistic determination of the Rouse time by use of Eqs. (9) or (14) is impossible. Therefore, we use $M_{\mathrm{cm}}$ in Eq. (15) as a fitting parameter for the elongational viscosity data. We follow Larson et al. (2003) here, who have justified the adjustment of $\tau_{e}$ (or equivalently $M_{\mathrm{cm}}$ in Eq. (15)) as a fitting parameter, and they mentioned that the adjustment of $\tau_{e}$ provides a strong test for tube models as this relaxation time must in principle remain constant at different molecular weights and chain architectures. As shown in the "Comparison between experimental data and model predictions" section for PtBS and PMMA, $\tau_{R}$ calculated by Eq. (15) with $M_{\mathrm{cm}}=3.2 M_{\mathrm{em}}$ and $M_{\mathrm{cm}}=1.6 M_{\mathrm{em}}$, respectively, gives the best fits for the elongational viscosity data. Fuchs et al. (1996) deduced a relation of $M_{\mathrm{cm}} \approx 2 M_{\mathrm{em}}$ for PMMAs of different tacticities, which is roughly in agreement with our relation for PMMA. The number $N_{e}$ of Kuhn segments or "monomers" per entanglement as reported by Morelly et al. (2019) is 29 for PtBS, 16 for PS and 7 or 8 for PMMA (Table 1), i.e. $N_{e}$ decreases in the sequence of PtBS, PS and PMMA. We note that there is a similar trend seen in the ratio of $M_{\mathrm{cm}} / M_{\mathrm{em}}$, which decreases from 3.2 for PtBS, to 2.6 for PS and to 1.6 for PMMA. $\tau_{R}$ calculated by Eq. (14) using $\tau_{c}$ is also given in Table 1. Considering the experimental difficulties in determining the exact value of $\tau_{c}$, which may also be affected by glassy modes, and taking into account the assumed equivalence of $\tau_{c}$ and $\tau_{e}$, the difference with the values calculated by Eq. (15) for PtBS, PMMA-86k and PMMA-100k is not surprising.

We also note that PMMA-86k has a plateau modulus of $G_{N}=720 \mathrm{kPa}$, while the plateau modulus of PMMA-100k is $G_{N}=850 \mathrm{kPa}$. As explained in Wagner et al. (2018), this difference is most likely due to the difference in the tacticity of two melts. As no information is available on the tacticity of the 
PMMA melts of this study, we can only refer to Fetters et al. (1999a) reporting a plateau modulus of $310 \mathrm{kPa}$ and of $760 \mathrm{kPa}$ for an atactic and a predominantly syndiotactic PMMA, respectively. Comparing the reported values of Fetters et al. (1999a) with the plateau moduli of PMMA-86k and PMMA-100k, it can be concluded that PMMA-100k has higher tacticity than PMMA-86k.

Table 1 shows the molar mass $(M)$, polydispersity index $\left(M / M_{n}\right)$, density $(\rho)$, glass transition temperature $\left(T_{g}\right)$, test temperature $(T)$, plateau modulus $\left(G_{N}\right)$, terminal/reorientational/ disengagement/reptation time $\left(\tau_{d}\right)$, zero-shear viscosity $\left(\eta_{0}\right)$, Rouse stretch relaxation time $\left(\tau_{R}\right)$ and number of entanglements per chain $(Z)$ of the monodisperse polymers melts considered in this study. The numbers of Kuhn segments or "monomers" per entanglement are taken from Morelly et al. (2019).

The discrete relaxation spectra of PtBS-310k at $T=175^{\circ} \mathrm{C}$, PMMA-86k at $T=150{ }^{\circ} \mathrm{C}$, PMMA-100k at $T=160^{\circ} \mathrm{C}$, PS200k at $T=130^{\circ} \mathrm{C}$ (Bach et al. 2003a) and PS-285k at $T=128$ ${ }^{\circ} \mathrm{C}$ (Huang et al. 2013a) are reported in Table 2.

\section{The extended interchain pressure model}

The extended interchain pressure (EIP) model was developed for extensional flow (Narimissa et al. 2020a) and shear flow (Narimissa et al. 2020b) of monodisperse linear polymer melts and solutions. The EIP model relaxes one of the basic assumptions of the original tube model of Doi and Edwards (1978a, b), i.e. the assumption of a constant tube diameter, and assumes that chain stretch $f$ is inverse proportional to a deformation-dependent tube diameter (see Narimissa et al. (2020a) for more details). The extra stress tensor of the EIP model is then given as

$\boldsymbol{\sigma}(t)=\int_{-\infty}^{t} \frac{\partial G\left(t-t^{\prime}\right)}{\partial t^{\prime}} f^{2} \mathbf{S}_{\mathrm{DE}}^{\mathrm{IA}}\left(t, t^{\prime}\right) d t^{\prime}$

where $G(t)$ is the linear viscoelastic shear relaxation modulus and the relative strain measure $\mathrm{S}_{\mathrm{DE}}^{\mathrm{IA}}$ is given by

$\mathbf{S}_{\mathrm{DE}}^{\mathrm{IA}}\left(t, t^{\prime}\right) \equiv 5\left\langle\frac{\mathbf{u}^{\prime} \mathbf{u}^{\prime}}{u^{\prime 2}}\right\rangle_{\mathrm{o}}=5 \mathbf{S}\left(t, t^{\prime}\right)$

$\mathbf{S}$ is the relative second-order orientation tensor. The bracket denotes an average over an isotropic distribution of unit vectors $\mathbf{u}\left(t^{\prime}\right)$ at time $t^{\prime}$ and can be expressed as a surface integral over the unit sphere,

$$
\langle\ldots\rangle_{\mathrm{o}} \equiv \frac{1}{4 \pi} \oiint[\ldots] \sin \theta_{o} d \theta_{o} d \varphi_{o}
$$

At the observation time $t$, the unit vectors are deformed to vectors $\mathbf{u}^{\prime}$, which are calculated from the affine deformation hypothesis (with $\mathbf{F}^{-1}\left(t, t^{\prime}\right)$ being the relative deformation gradient tensor) as

$\mathbf{u}^{\prime}\left(t, t^{\prime}\right)=\mathbf{F}^{-1}\left(t, t^{\prime}\right) \cdot \mathbf{u}\left(t^{\prime}\right)$

$u^{\prime}$ indicates the length of the vector $\mathbf{u}^{\prime}$.

The stretch evolution equation of the EIP model for polymer melts is given as (Wagner and Rolón-Garrido 2009a; Wagner and Rolón-Garrido 2009b; Narimissa et al. 2020a),

$\frac{\partial f}{\partial t}=f(\kappa: \mathbf{S})-\frac{1}{3} \frac{f-1}{\tau_{R}}-\frac{2}{3} \frac{f^{2}\left(f^{3}-1\right)}{3 \tau_{R}}$

The first term on the right-hand side represents an on average affine stretch rate with $\kappa$ the velocity gradient tensor, the second term takes into account Rouse relaxation with Rouse time $\tau_{R}$ according to Eq. (15) in the longitudinal direction of the tube and the third term limits molecular stretch due to the interchain tube pressure in the lateral direction of a tube segment.

For steady-state elongational flow $\left(\frac{\partial f}{\partial t}=0\right)$ and large stretch of melts, $f^{2}$ reaches a limiting value of

$f^{2} \cong \frac{3}{2} \sqrt{2 \tau_{R} \dot{\varepsilon}}$

Therefore, steady-state elongational stress $\sigma_{E}(\dot{\varepsilon})$ at sufficiently large elongation rates, i.e. for $W i=\tau_{R} \dot{\varepsilon}>>1$ from Eqs. (16) and (21), is given as,

$\sigma_{E}(W i)=\frac{15}{2} G_{N} \sqrt{2 W i}$

In the following section, we assess the modelling competence of the EIP model with extra stress tensor, Eq. (16), stretch evolution equation Eq. (20) and Rouse stretch relaxation time, Eq. (15), for the prediction of the uniaxial extensional flow data of PS-200k, PS-285k, PtBS-310k, PMMA86k and PMMA-100k.

\section{Comparison between experimental data and model predictions}

\section{Monodisperse polystyrene melts}

As per reference for the PtBS and PMMA melts, which are in the focus here, we shortly present in Fig. 1 and Fig. 2 the prediction of the elongational stress growth coefficient and steady-state elongational viscosity of PS-200k and PS-285k by the EIP model, i.e. Eqs. (15), (16) and (20). Excellent agreement between model predictions and experimental data 

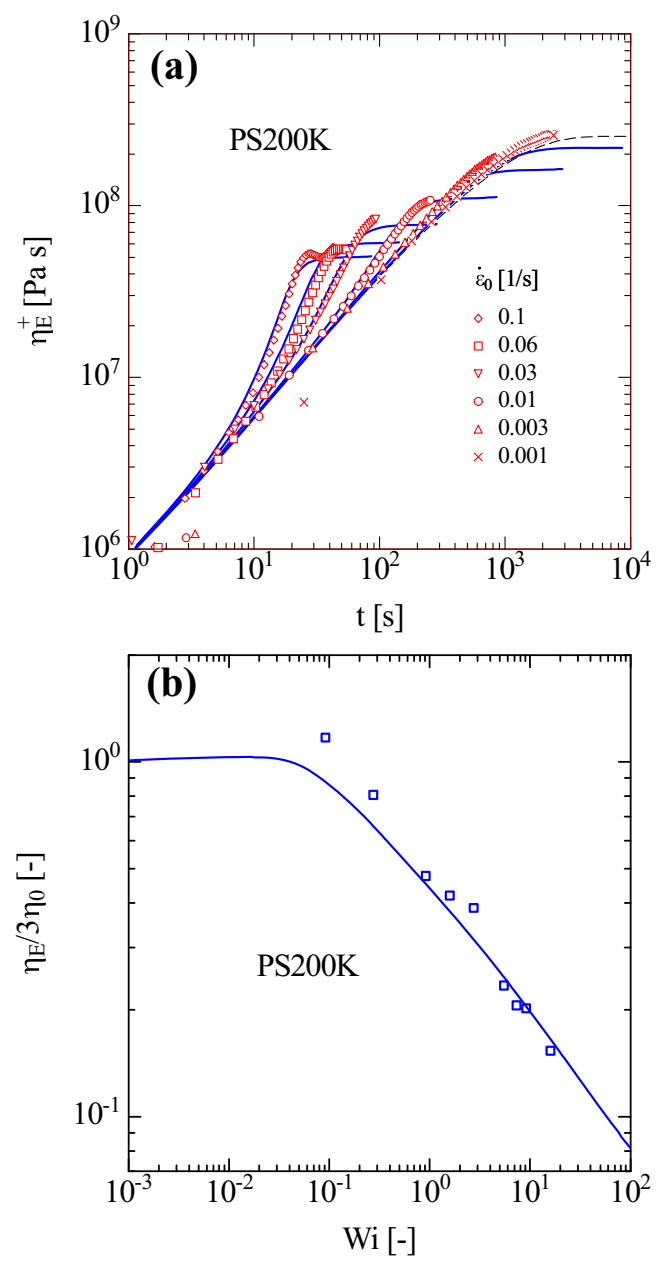

Fig. 1 Data (symbols) of elongational stress growth coefficient (a) and steady-state (b) elongational viscosity of PS-200k (refer to Table 1) and predictions (lines) by the EIP model, Eqs. (15), (16) and (20)

is achieved at all strain rates for the stress growth coefficient of PS-200k and PS-285k (Fig. 1a and Fig. 2a).

The prediction of the steady-state elongational viscosity of PS-200k at Weissenberg number larger than unity, i.e. $W i=\tau_{R} \dot{\varepsilon} \geq 1$, is in excellent agreement with the model, while slight underprediction is seen at $W i<1$ (Fig. 1b), which is well within the limits of experimental accuracy. Similarly, in the case of PS-285k, very good agreement between the EIP model and data is achieved at $W i \geq 0.03$ (Fig. 2b).

\section{Monodisperse poly(tert-butylstyrene) melt}

\section{Linear viscoelastic characterization}

The linear viscoelastic data of storage $\left(G^{\prime}\right)$ and loss $\left(G^{\prime \prime}\right)$ modulus of PtBS-301k at $175^{\circ} \mathrm{C}$ were reported by Morelly et al. (2019). Figure 3 shows the fit (red lines) of $G^{\prime}$ and $G^{\prime \prime}$ (symbols) through the BSW spectrum (Baumgaertel et al. 1990), Eqs. (1)-(4). The BSW fit underpredicts both storage $\mathrm{G}^{\prime}$ and loss $\mathrm{G}^{\prime \prime}$ modulus. The fit by the parsimonious spectrum
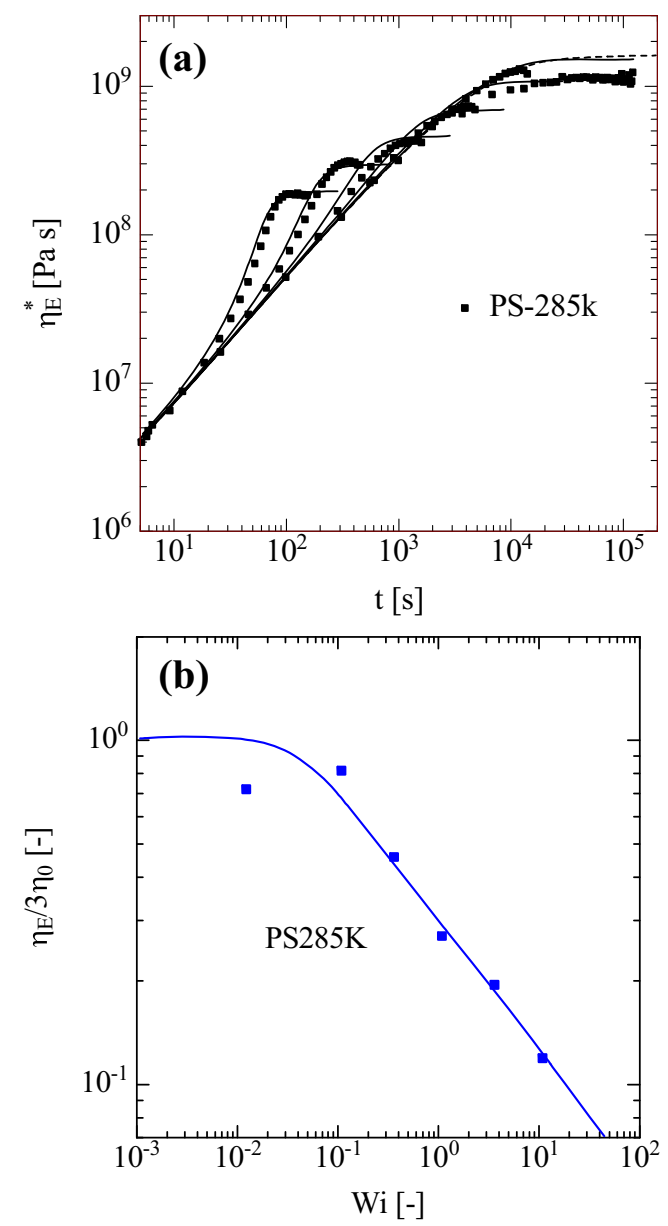

Fig. 2 Data (symbols) of elongational stress growth coefficient (a) and steady-state (b) elongational viscosity of PS-285k (refer to Table 1) and predictions (lines) by the EIP model, Eqs. (15), (16) and (20)

(blue lines) in the experimentally accessible frequency window using the IRIS software (Winter and Mours 2006) goes directly through the data points and is in excellent agreement with the linear viscoelastic data. Therefore, we use the discrete relaxation spectrum (Table 2) for the prediction of elongational flow behaviours of PtBS-301k. The exact representation of the LVE behaviour is essential for the assessment of linear and nonlinear rheological universality.

\section{Elongational flows}

Comparison of EIP model predictions (lines) and data of the elongational stress growth coefficient and the steady-state elongational viscosity (symbols) of PtBS-301k at $175^{\circ} \mathrm{C}$ are shown in Fig. 4. We note that the elongational stress growth coefficients at $\dot{\varepsilon}=5 \times 10^{-5} \mathrm{~s}^{-1}$ and $\dot{\varepsilon}=3.73 \times 10^{-3} \mathrm{~s}^{-1}$ were actually measured at $200{ }^{\circ} \mathrm{C}$ and time temperature shifted to $175^{\circ} \mathrm{C}$. This may be the reason why some deviation is seen between experimental data and prediction at $\dot{\varepsilon}=3.73 \times 10^{-3} \mathrm{~s}^{-1}$; otherwise, general agreement 


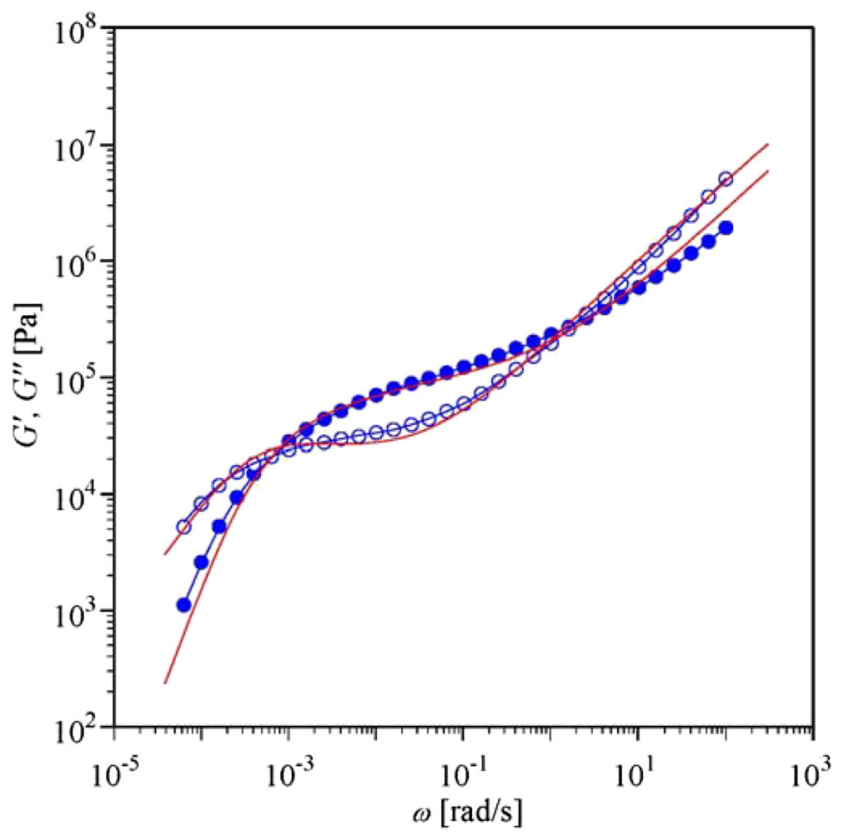

Fig. 3 BSW fit (red lines), Eqs. (1)-(4), and fit (blue lines) by discrete relaxation spectrum (Table 2) for storage $\left(G^{\prime}\right)$ and loss $\left(G^{\prime \prime}\right)$ modulus of PtBS-301k at $175^{\circ} \mathrm{C}$

between data and model within experimental accuracy is seen. As discussed in the "LVE characterization" section, the Rouse time $\tau_{R}$ calculated by Eq. (15) with $M_{\mathrm{cm}}=3.2 M_{\mathrm{em}}$ gives the best fit for the elongational viscosity data.

\section{Monodisperse poly(methyl-methacrylate) melts}

\section{Linear viscoelastic characterization}

Figure 5 and Fig. 6 show the linear viscoelastic storage $\left(G^{\prime}\right)$ and loss $\left(G^{\prime \prime}\right)$ modulus of PMMA-86k and PMMA-100k at the testing temperatures of $150{ }^{\circ} \mathrm{C}$ and $160{ }^{\circ} \mathrm{C}$, respectively. Similar to PtBS-301k (Fig. 3), BSW fits of the relaxation spectra (red lines) resulted in qualitative agreement with storage and loss modulus data of the PMMA melts, while fits of the data by parsimonious relaxation spectra (Table 2) are in perfect quantitative agreement. Hence, we use the discrete relaxation spectra for the prediction of elongational flow behaviours of PMMA-86k and PMMA-100k.

\section{Elongational flow}

Figure 7 shows the comparison between the experimental data and prediction of the elongational flow behaviour of PMMA$86 \mathrm{k}$ by the EIP model. As discussed in the "LVE characterization" section, the Rouse time $\tau_{R}$ calculated by Eq. (15) with $M_{\mathrm{cm}}=1.6 M_{\mathrm{em}}$ gives the best fit for the elongational viscosity. The start-up regimes of the stress growth coefficient are in quantitative agreement with the model, as well as the steady-
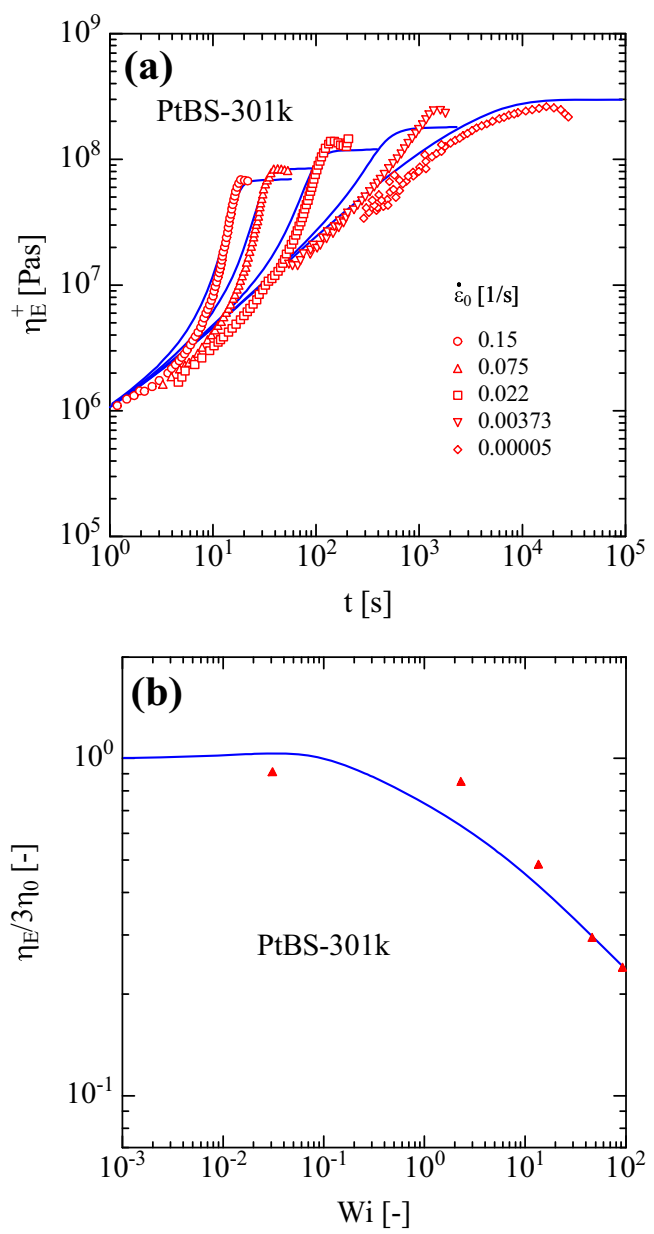

Fig. 4 Data (symbols) of elongational stress growth coefficient (a) and steady-state (b) elongational viscosity of PtBS-301k (refer to Table 1) and predictions (lines) by the EIP model, Eqs. (15), (16) and (20)

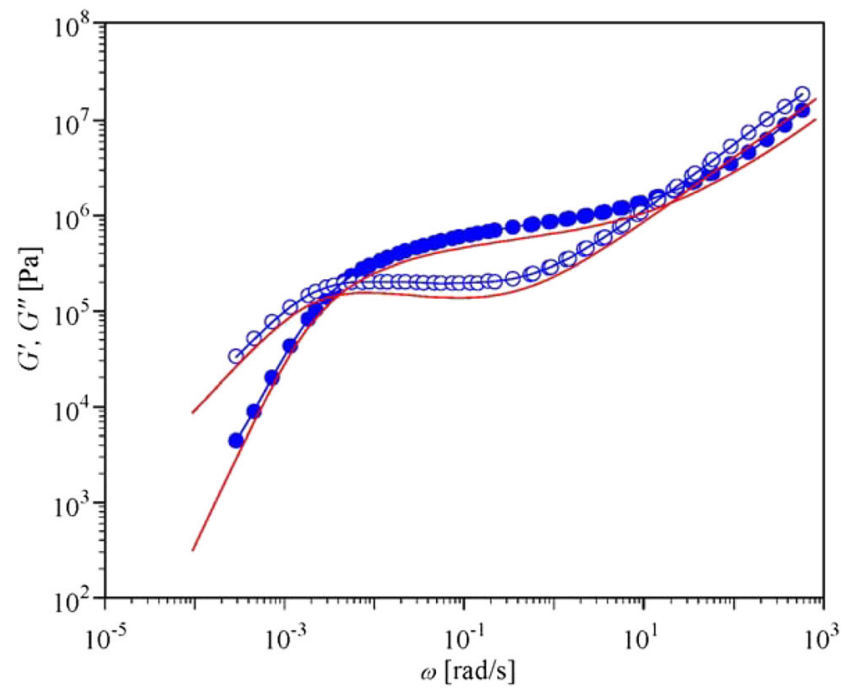

Fig. 5 BSW fit (red lines), Eqs. (1)-(4), and fit (blue lines) by discrete relaxation spectrum (Table 2) for storage $\left(G^{\prime}\right)$ and loss $\left(G^{\prime \prime}\right)$ modulus of PMMA-86k at $150{ }^{\circ} \mathrm{C}$ 


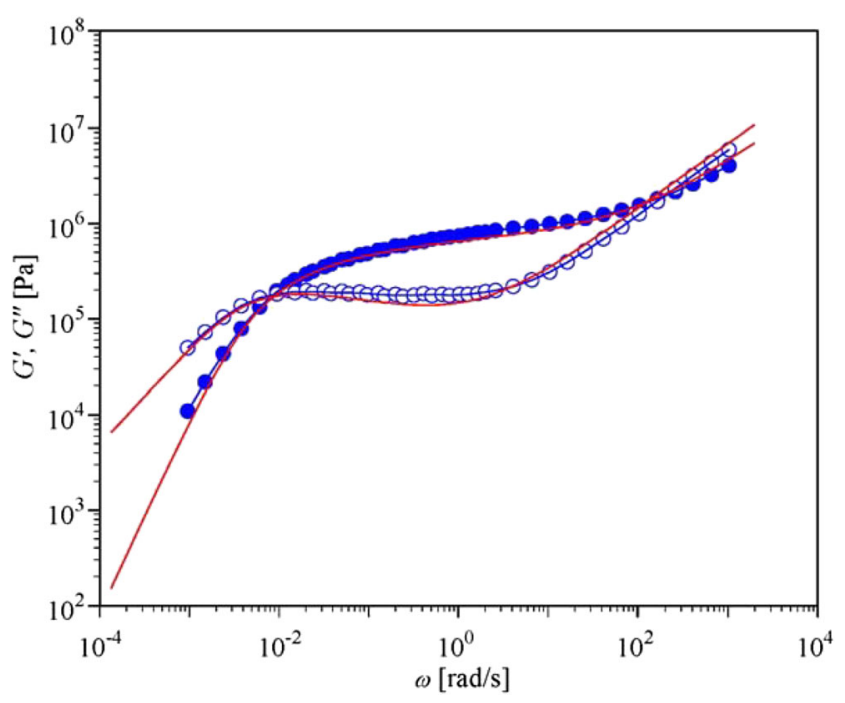

Fig. 6 BSW fit (red lines), Eqs. (1)-(4), and fit (blue lines) by discrete relaxation spectrum (Table 2) for storage $\left(G^{\prime}\right)$ and loss $\left(G^{\prime \prime}\right)$ modulus of PMMA-100k at $160{ }^{\circ} \mathrm{C}$
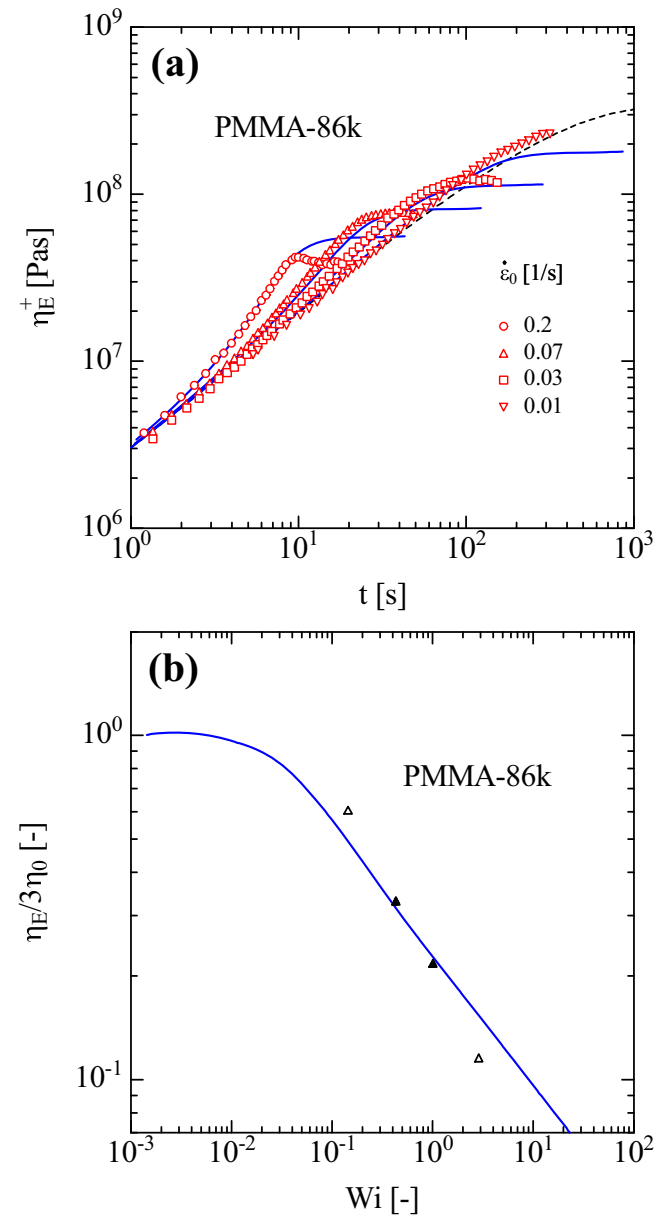

Fig. 7 Data (symbols) of elongational stress growth coefficient (a) and steady-state (b) elongational viscosity of PMMA-86k (refer to Table 1) and predictions (lines) by the EIP model, Eqs. (15), (16) and (20) state viscosity at the strain rates of $\dot{\varepsilon}=0.03 \mathrm{~s}^{-1}$ and $\dot{\varepsilon}=0.07 \mathrm{~s}^{-1}$. For the lowest $\left(0.01 \mathrm{~s}^{-1}\right)$ and the highest strain rate $\left(0.2 \mathrm{~s}^{-1}\right)$, clearly no steady-state is achieved experimentally (indicated in Fig. 7b by open symbols). At the strain rate of $0.2 \mathrm{~s}^{-1}$, significant overshoot is observed experimentally (Fig. 7a). Overshoot can be either the result of a material property of the polymer (Burghelea et al. 2011; Narimissa and Wagner 2019; Rasmussen and Fasano 2018) where, in the case of long-chain branched polymers, it can be caused by the disentanglement of dangling arms after branch point withdrawal and reversion to the behaviour of linear polymers (Wagner and Rolón-Garrido 2008) or it can be the result of non-uniformity of sample deformation at high strains. As for linear PMMA, no overshoot of the elongational stress growth coefficient is expected; we conclude that the overshoot observed is most likely caused by the non-uniform deformation.

In the case of PMMA-100k (Fig. 8), the EIP model again with the Rouse time $\tau_{R}$ calculated by Eq. (15) with $M_{\mathrm{cm}}=$ $1.6 M_{\mathrm{em}}$ is in good agreement with the start-up of the stress growth coefficient. However, it seems that with the exception of the highest strain rate $\left(0.6 \mathrm{~s}^{-1}\right)$, for which excellent agreement between the steady-state elongational viscosity and model prediction is seen, no steady-state viscosity was reached experimentally. Therefore, a comparison of steadystate elongational viscosity data and model predictions is not possible in the case of PMMA-100k.

\section{Discussion and conclusions}

We compare the elongational steady-state behaviours of the polymer melts considered here and test the conjecture of Morelly et al. (2019) about the independence of the elongational stress on the number of entanglements $(Z)$ as opposed to the dependence of the elongational viscosity on $Z$.

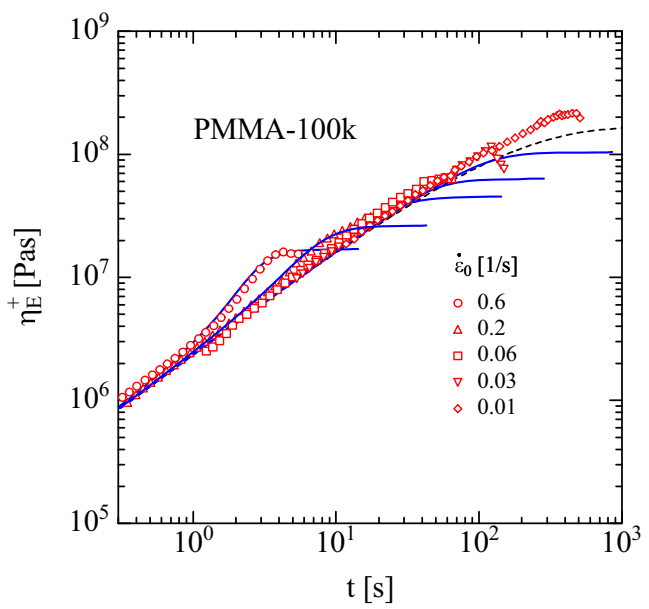

Fig. 8 Data (symbols) of elongational stress growth coefficient of PMMA-100k (refer to Table 1) and predictions (lines) by the EIP model, Eqs. (15), (16) and (20) 
As shown by Eq. (22), in the limit of fast elongational flow (high $W i$ ), the normalized steady-state elongational stress of melts is proportional to the square root of the Weissenberg number,

$\frac{\sigma_{E}(W i)}{G_{N}}=\frac{15}{2} \sqrt{2 W i}$

Figure 9za shows the normalized steady-state elongational stress as a function of Wi. The green asymptote in Fig. 9a, showing a slope of $1 / 2$, is given by Eq. (23). The slope of $\sigma_{E} /$ $G_{N}$ changes from 1 in the linear viscoelastic regime $(W i<<1)$ to $1 / 2$ at high $W i$. However, this transition is quite gradual meaning that the effective slope in the experimentally accessible nonlinear viscoelastic regime is greater than $1 / 2$, and we observe a tendency that melts with higher values of $\sigma_{E} / G_{N}$ approach the limiting behaviour of Eq. (23) faster and have a lower slope. If this behaviour is approximated by a power law, the exponent increases in the sequence of PMMA, PS and
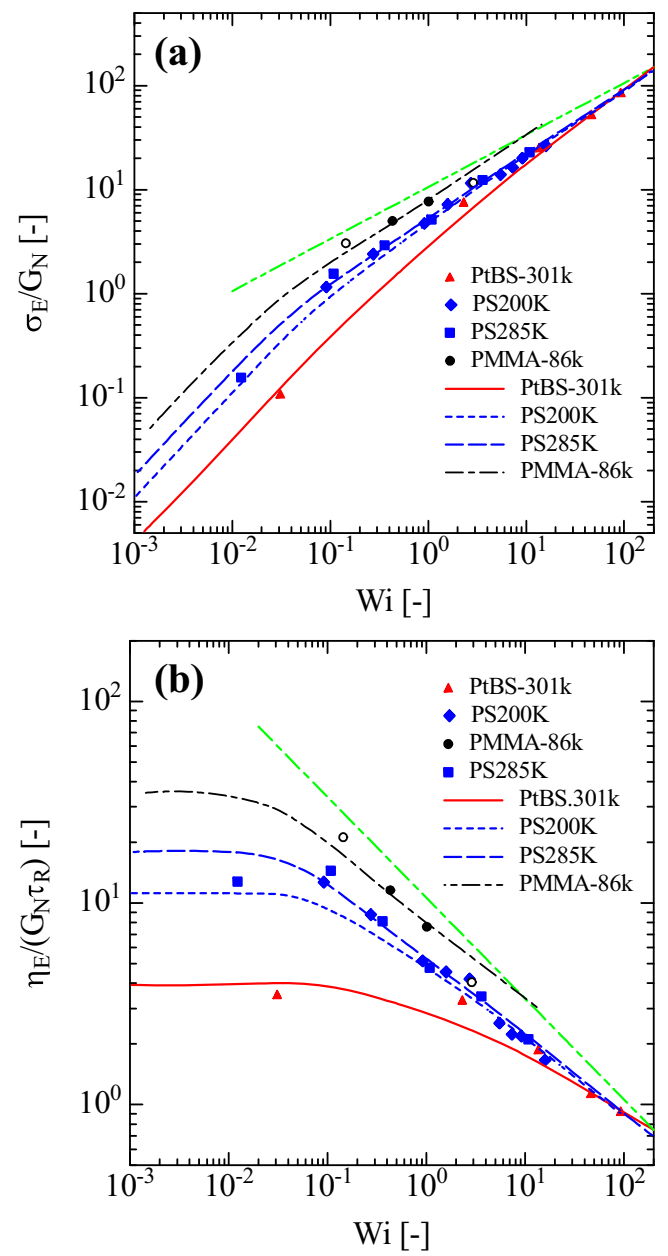

Fig. 9 Normalized elongational stress (a) and normalized elongational viscosity (b) as functions of Weissenberg number $W i=\tau_{R} \dot{\varepsilon}$ of PS-200k, PS-285k, PtBS-301k and PMMA-86k (symbols) and predictions (lines) via the EIP model, Eqs. (15), (16) and (20). The green dash-dotted asymptotes show a slope of $1 / 2$ in $\mathbf{a}$ and $-1 / 2$ in $\mathbf{b}$
PtBS. This explains the different power law exponents reported by Morelly et al. (see Fig. 8a in Morelly et al. (2019)), namely exponent of 0.4 for PMMA, 0.6 for PS and 0.7 for PtBS. Morelly et al. (2019) discussed the conjecture that the exponent may be related to the finite extensibility parameter $\lambda_{\max }$ but concluded that there appears to be no consistent scaling with $\lambda_{\text {max }}^{2} \cong N_{e}$, in agreement with the results of concentrated polymer solutions where no scaling of elongational stress with $\lambda_{\max }$ was observed. Following our earlier analysis of the elongational flow behaviours of polystyrene melts and solutions (Narimissa et al. 2020a), we do not consider finite extensibility here. However, as noted above, by fitting the Rouse times of PtBS and PMMA to the elongational viscosity data, we find a trend that the ratio of $M_{\mathrm{cm}} / M_{\mathrm{em}}$ according to Eqs. (5) and (15) decreases with decreasing number $N_{e}$ of Kuhn monomers in the sequence of PtBS, PS and PMMA.

Figure $9 \mathrm{~b}$ shows the elongational viscosity, normalized by $G_{N} \tau_{R}$, as a function of $W i$. Considering the relation between elongational stress and viscosity, $\eta_{E}=\sigma_{E} / \dot{\varepsilon}$, and the Hencky strain rate in terms of Weissenberg number, $\dot{\varepsilon}=W i / \tau_{R}$, the normalized viscosity at sufficiently large elongation rates becomes from Eq. (23),

$\frac{\eta_{E}(W i)}{G_{N} \tau_{R}}=\frac{15}{2} \sqrt{2} W i^{-1 / 2}$

The green asymptote in Fig. $9 \mathrm{~b}$ showing a slope of $-1 / 2$ is given by Eq. (24). To better understand the elongational viscosity behaviour of the polymer melts and the relationship with the molar mass, we note that in the LVE regime, the normalized zero-elongational viscosity $\eta_{E}^{0}=3 \eta_{0}$ (following from Eqs. (5) and (15)) is given by,

$\frac{\eta_{E}^{0}(M)}{G_{N} \tau_{R}}=\frac{\pi^{2}}{4}\left(\frac{M_{\mathrm{em}}}{M_{\mathrm{cm}}}\right)^{2.4}\left(\frac{M}{M_{\mathrm{em}}}\right)^{1.4}$

At constant ratio $M_{\mathrm{em}} / M_{\mathrm{cm}}$, the normalized zeroelongational viscosity increases with $\left(M / M_{\mathrm{em}}\right)^{1.4}=Z^{1.4}$, for example the normalized zero-elongational viscosity of PS285K with $Z=21.4$ is larger than of PS200K with $Z=$ 14.9 (Fig. 9b). On the other hand, at constant $Z$, the normalized zero-elongational viscosity increases with increasing ratio $M_{\mathrm{em}} / M_{\mathrm{cm}}$ according to $\left(M_{\mathrm{em}} / M_{\mathrm{cm}}\right)^{2.4}$, as seen in the difference of the normalized viscosities of PS200K $\left(Z=14.9, M_{\mathrm{em}} /\right.$ $\left.M_{\mathrm{cm}}=0.38\right)$ and PMMA-86k $\left(Z=14.9, M_{\mathrm{em}} / M_{\mathrm{cm}}=0.63\right)$ in Fig. $9 \mathrm{~b}$. It is evident that the slope of the reduced viscosity changes from 0 in the LVE regime to $-1 / 2$ at very high $W i$. However, as in the case of the elongational stress, this transition is quite gradual as already noted by Wagner et al. (2005), which means that the effective slope in the experimentally accessible nonlinear viscoelastic regime is larger than $-1 / 2$. There is a tendency that melts with a low value of the normalized zero-elongational viscosity such as PtBS have a higher 
slope in the nonlinear viscoelastic regime and show less strain thinning than melts with a large normalized viscosity such as PMMA-86k. This explains the differences seen in the $\eta_{E}(W i)$ behaviour of the melts as reported by Morelly et al. (see Fig. $8 \mathrm{~b}$ in Morelly et al. (2019)).

In summary, in the LVE regime of elongational flow, both stress and viscosity are dependent on the number of entanglements and the ratio between entanglement molar mass and critical molar mass, i.e. $\eta_{E}^{0} /\left(G_{N} \tau_{R}\right) \propto\left(M_{\mathrm{em}} / M_{\mathrm{cm}}\right){ }^{2.4} Z^{1.4}$ and $\sigma_{E}^{0} / G_{N} \propto\left(M_{\mathrm{em}} / M_{\mathrm{cm}}\right){ }^{2.4} Z^{1.4} W i$, while in the limit of fast elongational flow (high $W i$ ), both stress and viscosity become independent of $Z$ and $M_{\mathrm{em}} / M_{\mathrm{cm}}$, and approach a scaling relation which depends only on $W i$, i.e. $\eta_{E} /\left(G_{N} \tau_{R}\right) \propto W i^{-1 / 2}$ and $\sigma_{E} /$ $G_{N} \propto W i^{1 / 2}$. However, this universal scaling is only reached at very high Weissenberg numbers. When expressed by an effective power law, the broad transition from the linear viscoelastic to the high $W i$ regime leads to chemistry-dependent scaling at intermediate $W i$ with exponents depending on the number of entanglements and the ratio between entanglement molar mass and critical molar mass. We observe a tendency that melts with a low value of $\eta_{E}^{0} /\left(G_{N} \tau_{R}\right)$ feature a higher power law exponent than melts with a large value of the normalized zero-elongational viscosity. While the dependence on the number of entanglements is in agreement with the universality of the linear viscoelastic behaviour (i.e. melts with the same $Z$ show the same linear and nonlinear viscoelastic behaviour), the dependence of the elongational viscosity on the ratio between entanglement molar mass and critical molar mass seems surprising at first sight. However, as noted in the "Introduction" section, Fetters et al. (1999b) showed that $M_{\mathrm{em}} / M_{\mathrm{cm}}$ is chemistry dependent and concluded that the degree of entanglement, as specified by $M_{e m}$, is not sufficient to fully characterize the entanglement effects. Thus, the three material parameters, i.e. plateau modulus, characteristic time and number of entanglements, may not be sufficient to fully characterize the apparent universality of the linear viscoelastic behaviour of well-entangled monodisperse linear polymer melts and solutions, and this deficiency is replicated in the nonlinear viscoelastic regime. Further experimental evidence of polymers with different chemical constituents is required to confirm these conclusions.

Funding Open Access funding enabled and organized by Projekt DEAL.

Open Access This article is licensed under a Creative Commons Attribution 4.0 International License, which permits use, sharing, adaptation, distribution and reproduction in any medium or format, as long as you give appropriate credit to the original author(s) and the source, provide a link to the Creative Commons licence, and indicate if changes were made. The images or other third party material in this article are included in the article's Creative Commons licence, unless indicated otherwise in a credit line to the material. If material is not included in the article's Creative Commons licence and your intended use is not permitted by statutory regulation or exceeds the permitted use, you will need to obtain permission directly from the copyright holder. To view a copy of this licence, visit http://creativecommons.org/licenses/by/4.0/.

\section{References}

Bach A, Almdal K, Rasmussen HK, Hassager O (2003a) Elongational viscosity of narrow molar mass distribution polystyrene. Macromolecules 36:5174-5179

Bach A, Rasmussen HK, Hassager O (2003b) Extensional viscosity for polymer melts measured in the filament stretching rheometer. $\mathrm{J}$ Rheol 47:429-441. https://doi.org/10.1122/1.1545072

Baumgaertel M, Schausberger A, Winter HH (1990) The relaxation of polymers with linear flexible chains of uniform length. Rheol Acta 29:400-408

Bhattacharjee PK, Oberhauser JP, McKinley GH, Leal LG, Sridhar T (2002) Extensional rheometry of entangled solutions. Macromolecules 35: 10131-10148. DOI Export Date 24 August 2013

Burghelea TI, Starý Z, Münstedt H (2011) On the "viscosity overshoot" during the uniaxial extension of a low density polyethylene. J NonNewtonian Fluid Mech 166: 1198-1209. DOI Export Date 24 August 2013

Daoud M, De Gennes P (1979) Some remarks on the dynamics of polymer melts. J Poly Sci: Polymer Physics Edition 17:1971-1981

Dealy JM, Read DJ, Larson RG (2018) Structure and rheology of molten polymers: from structure to flow behavior and back again. Carl Hanser Verlag GmbH Co KG

Desai PS, Larson RG (2014) Constitutive model that shows extension thickening for entangled solutions and extension thinning for melts. J Rheol 58:255-279

Doi M (1981) Explanation for the 3.4 power law of viscosity of polymeric liquids on the basis of the tube model. J Poly Sci: Polymer Letters Edition 19:265-273

Doi M, Edwards SF (1978a) Dynamics of concentrated polymer systems. Part 2. - Molecular motion under flow. J Chem Soc, Faraday Transactions 2: Molecular and Chemical Physics 74: 1802-1817

Doi M, Edwards SF (1978b) Dynamics of concentrated polymer systems. Part 3. - The constitutive equation. J Chem Soc, Faraday Transactions 2: Molecular and Chemical Physics 74: 1818-1832

Doi M, Edwards SF (1979) Dynamics of concentrated polymer systems. Part 4.-Rheological properties. Journal of the Chemical Society, Faraday Transactions 2: Molecular and Chemical Physics 75: 38-54. DOI https://doi.org/10.1039/F29797500038

Doi M, Edwards SF (1986) The theory of polymer dynamics. Oxford University Press, Oxford

Ferry JD (1980) Viscoelastic properties of polymers. John Wiley and Sons, New York

Fetters LJ, Lohse DJ, Graessley WW (1999a) Chain dimensions and entanglement spacings in dense macromolecular systems. J Polym Sci Part B: Polymer Physics 37:1023-1033

Fetters LJ, Lohse DJ, Milner ST, Graessley WW (1999b) Packing length influence in linear polymer melts on the entanglement, critical, and reptation molecular weights. Macromolecules 32:6847-6851

Flory PJ (1953) Principles of polymer chemistry. Cornell University Press, New York

Fuchs K, Friedrich C, Weese J (1996) Viscoelastic properties of narrowdistribution poly (methyl methacrylates). Macromolecules 29:58935901

Gennes PG (1971) Reptation of a polymer chain in the presence of fixed obstacles. The Journal of Chemical Physics 55:572-579. https://doi. org/10.1063/1.1675789 
Huang Q, Alvarez NJ, Matsumiya Y, Rasmussen HK, Watanabe H, Hassager O (2013a) Extensional rheology of entangled polystyrene solutions suggests importance of nematic interactions. ACS Macro Letters 2:741-744

Huang Q, Mednova O, Rasmussen HK, Alvarez NJ, Skov AL, Almdal K, Hassager O (2013b) Concentrated polymer solutions are different from melts: role of entanglement molecular weight. Macromolecules 46:5026-5035

Huang Q, Hengeller L, Alvarez NJ, Hassager O (2015) Bridging the gap between polymer melts and solutions in extensional rheology. Macromolecules 48:4158-4163

Ianniruberto G (2015) Extensional flows of solutions of entangled polymers confirm reduction of friction coefficient. Macromolecules 48: 6306-6312

Ianniruberto G, Marrucci G (1996) On compatibility of the Cox-Merz rule with the model of Doi and Edwards. J Non-Newtonian Fluid Mech 65:241-246. https://doi.org/10.1016/0377-0257(96)01433-4

Ianniruberto G, Brasiello A, Marrucci G (2012) Simulations of fast shear flows of PS oligomers confirm monomeric friction reduction in fast elongational flows of monodisperse PS melts as indicated by rheooptical data. Macromolecules 45:8058-8066

Ianniruberto G, Marrucci G, Masubuchi Y (2020) Melts of linear polymers in fast flows. Macromolecules 53:5023-5033

Isaki T, Takahashi M, Urakawa O (2003) Biaxial damping function of entangled monodisperse polystyrene melts: comparison with the Mead-Larson-Doi model. J Rheol 47: 1201-1210. DOI Export Date 24 August 2013

Kuhn W (1934) Über die Gestalt fadenförmiger Moleküle in Lösungen. Kolloid-Zeitschrift 68:2-15. https://doi.org/10.1007/BF01451681

Larson R, Sridhar T, Leal L, McKinley GH, Likhtman A, McLeish T (2003) Definitions of entanglement spacing and time constants in the tube model. Journal of Rheology 47:809-818

Luap C, Müller C, Schweizer T, Venerus DC (2005) Simultaneous stress and birefringence measurements during uniaxial elongation of polystyrene melts with narrow molecular weight distribution. Rheol Acta 45:83-91

Marrucci G, Grizzuti N (1988) Fast flows of concentrated polymers: predictions of the tube model on chain stretching. Gazz Chim Itali 118:179-185

Marrucci G, Ianniruberto G (2004) Interchain pressure effect in extensional flows of entangled polymer melts. Macromolecules 37:3934 3942

Menezes E, Graessley W (1982) Nonlinear rheological behavior of polymer systems for several shear-flow histories. J Poly Sci Part B: Poly Phys 20:1817-1833

Morelly SL, Palmese L, Watanabe H, Alvarez NJ (2019) Effect of finite extensibility on nonlinear extensional rheology of polymer melts. Macromolecules 52:915-922

Narimissa E, Wagner MH (2019) Review on tube model based constitutive equations for polydisperse linear and long-chain branched polymer melts. J Rheol 63:361-375. https://doi.org/10.1122/1.5064642

Narimissa E, Huang Q, Wagner MH (2020a) Elongational rheology of polystyrene melts and solutions: concentration dependence of the interchain tube pressure effect. J Rheol 64:95-110. https://doi.org/ $10.1122 / 1.5100671$
Narimissa E, Schweizer T, Wagner MH (2020b) A constitutive analysis of nonlinear shear flow. Rheologica Acta 59:487-506. https://doi. org/10.1007/s00397-020-01215-7

Osaki K, Nishizawa K, Kurata M (1982) Material time constant characterizing the nonlinear viscoelasticity of entangled polymeric systems. Macromolecules 15: 1068-1071. DOI Export Date 24 August 2013

Park GW, Ianniruberto G (2017) Flow-induced nematic interaction and friction reduction successfully describe ps melt and solution data in extension startup and relaxation. Macromolecules 50:4787-4796

Rasmussen HK, Fasano A (2018) Flow and breakup in extension of lowdensity polyethylene. Rheol Acta 57:317-325

Rouse PE Jr (1953) A theory of the linear viscoelastic properties of dilute solutions of coiling polymers. J Chem Phys 21:1272-1280

Takahashi M, Isaki T, Takigawa T, Masuda T (1993) Measurement of biaxial and uniaxial extensional flow behavior of polymer melts at constant strain rates. J Rheol 37:827-846. https://doi.org/10.1122/1. 550397

Wagner MH (2014) Scaling relations for elongational flow of polystyrene melts and concentrated solutions of polystyrene in oligomeric styrene. Rheol Acta 53:765-777. https://doi.org/10.1007/s00397-0140791-1

Wagner MH, Rolón-Garrido VH (2008) Verification of branch point withdrawal in elongational flow of pom-pom polystyrene melt. J Rheol 52:1049-1068. https://doi.org/10.1122/1.2957699

Wagner MH, Rolón-Garrido VH (2009a) Nonlinear rheology of linear polymer melts: modeling chain stretch by interchain tube pressure and Rouse time. Korea Australia Rheol J 21:203-211

Wagner MH, Rolón-Garrido VH (2009b) Recent advances in constitutive modeling of polymer melts. novel trends of rheology III. AIP Conf Proc 1152:16-31. https://doi.org/10.1063/1.3203266

Wagner MH, Kheirandish S, Hassager O (2005) Quantitative prediction of transient and steady-state elongational viscosity of nearly monodisperse polystyrene melts. J Rheol 49:1317-1327

Wagner MH, Wingstrandt SL, Alvarez NJ, Narimissa E (2018) The peculiar elongational viscosity of concentrated solutions of monodisperse PMMA in oligomeric MMA. Rheologica Acta 57:591-601. https://doi.org/10.1007/s00397-018-1098-4

Winter HH, Mours M (2006) The cyber infrastructure initiative for rheology. Rheol Acta 45:331-338

Yaoita T, Isaki T, Masubuchi Y, Watanabe H, Ianniruberto G, Marrucci G (2012) Primitive chain network simulation of elongational flows of entangled linear chains: stretch/orientation-induced reduction of monomeric friction. Macromolecules 45:2773-2782

Ye X, Larson RG, Pattamaprom C, Sridhar T (2003) Extensional properties of monodisperse and bidisperse polystyrene solutions. J Rheol 47:443-468

Zimm BH (1956) Dynamics of polymer molecules in dilute solution: viscoelasticity, flow birefringence and dielectric loss. The Journal of Chemical Physics 24:269-278. https://doi.org/10.1063/1. 1742462

Publisher's note Springer Nature remains neutral with regard to jurisdictional claims in published maps and institutional affiliations. 